\title{
The diagnostic accuracy of OCT angiography in naive and treated neovascular age-related macular degeneration: a review
}

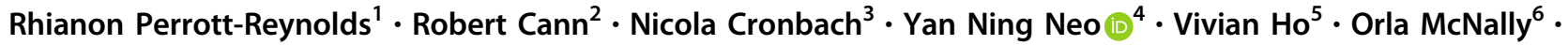 \\ Haifa A Madi ${ }^{7}$. Claire Cochran ${ }^{8} \cdot$ Usha Chakravarthy ${ }^{9,10}$
}

Received: 1 April 2018 / Revised: 10 July 2018 / Accepted: 24 August 2018 / Published online: 31 October 2018

(c) The Royal College of Ophthalmologists 2018

\begin{abstract}
Optical coherence tomography angiography (OCTA) is a non-invasive retinal imaging innovation that has been gaining popularity for the evaluation of the retinal vasculature. Of clinical importance is its current use either as an alternative or in conjunction with conventional dye-based angiography in neovascular age-related macular degeneration. OCTA is not without limitations and these include image artefact, a relatively small field of view and failure of the segmentation algorithms, which can confound the interpretation of findings. While there are numerous publications on OCTA in neovascular AMD, few have examined the diagnostic accuracy of this new technology compared with the accepted gold standard of fundus fluorescein angiography (FFA). In this review, we summarise the literature on the clinical application of OCTA in nAMD. In particular, we have reviewed the published articles that have reported the sensitivity and specificity of OCTA in the diagnosis of nAMD, and those that have described and or correlated the morphological findings and compared them to dye-based angiography.
\end{abstract}

\section{Introduction}

Optical coherence tomography angiography (OCTA) has gained enormous popularity since its introduction into the commercial sector in recent years [1,2]. Its main advantages in comparison to traditional techniques for visualisation of the posterior pole vasculature include the ease of image capture, rapid processing of the digital information and the high-resolution display of the retinal and choroidal vasculature profiles without the use of intravenous contrast

All authors are members of the EDNA Co-PI Writing Committee.

Rhianon Perrott-Reynolds

Rhianon.reynolds@wales.nhs.uk

1 Department of Ophthalmology, University Hospital of Wales, Cardiff CF14 4XW, UK

2 Mid Yorkshire Hospitals NHS Trust, West Yorkshire WF1 4DG, UK

3 Buckinghamshire Healthcare NHS trust, Oxford, UK

4 The Hillingdon Hospitals NHS Trust, Uxbridge UB8 3NN, UK

5 Royal Liverpool and Broadgreen University Hospitals NHS Trust, agents and dyes $[1,2]$. These features of OCTA imaging have overcome some of the challenges and risks, albeit small, of dye-based angiography, such as the need for cannulation and administration of intravenous substances. Acquisition of the OCTA image is quick and the processing immediate, and thus information on the retinal and choroidal circulation can be obtained almost immediately and with greater resolution than possible with traditional dyebased angiography $[1,2]$.

Despite these obvious advantages of OCTA, the segmentation of the individual layer boundaries, which is critical for displaying high-resolution images of the vascular profiles, can fail. Therefore, there are concerns relating to the use of OCTA alone to diagnose neovascular age-related

Liverpoool L14 3LB, UK

6 Belfast Health and Social Care Trust, Belfast BT9 7AB, UK

7 Sunderland Eye Infirmary, Sunderland SR2 9HP, UK

8 EDNA Study Team, University of Aberdeen, Aberdeen AB25 2ZD, UK

9 Ophthalmology and Vision Science, Queens University of Belfast, Belfast BT7 1NN, UK

10 Department of Ophthalmology, Belfast Health and Social Care Trust, Belfast BT9 7AB, UK 


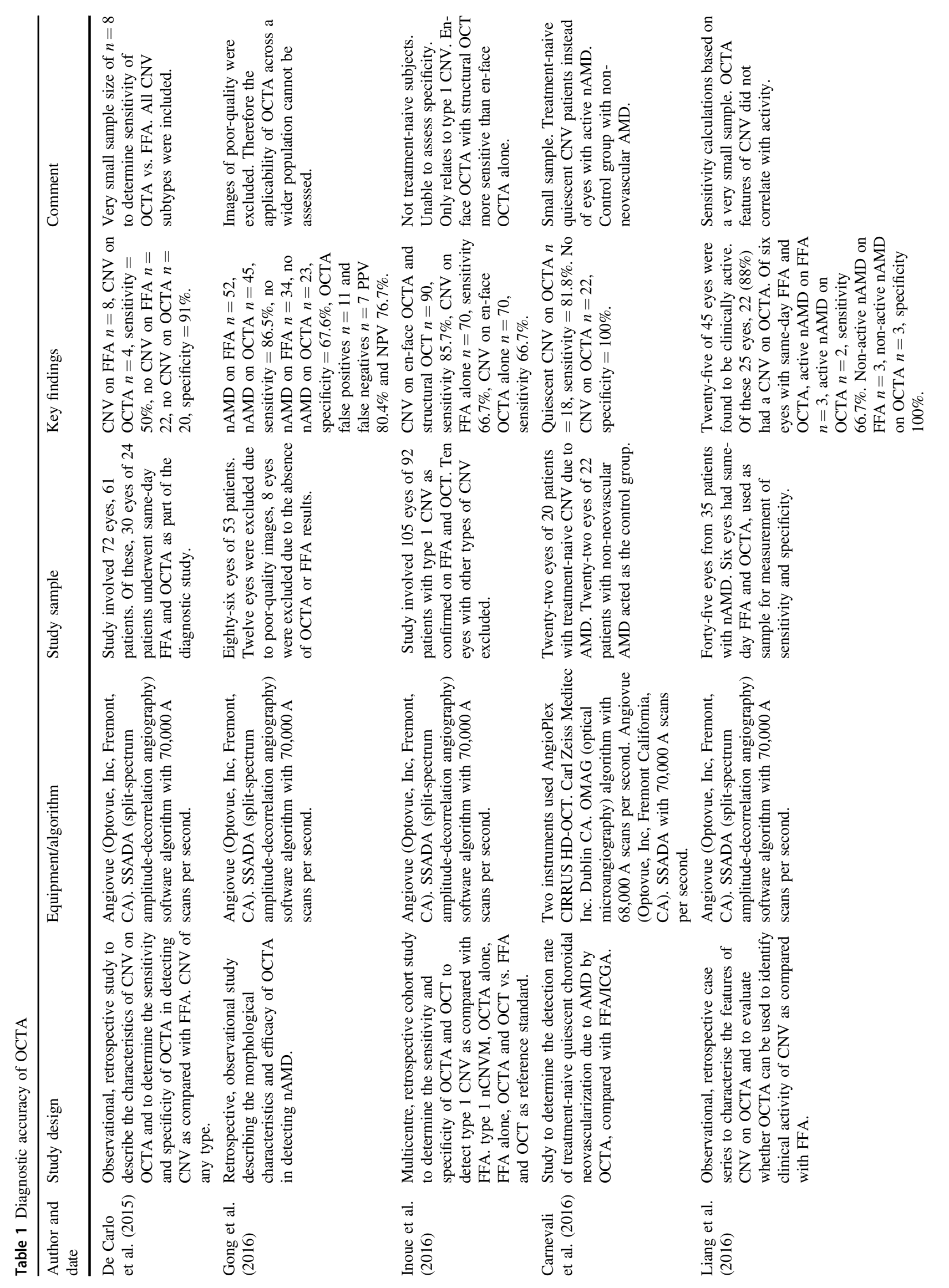




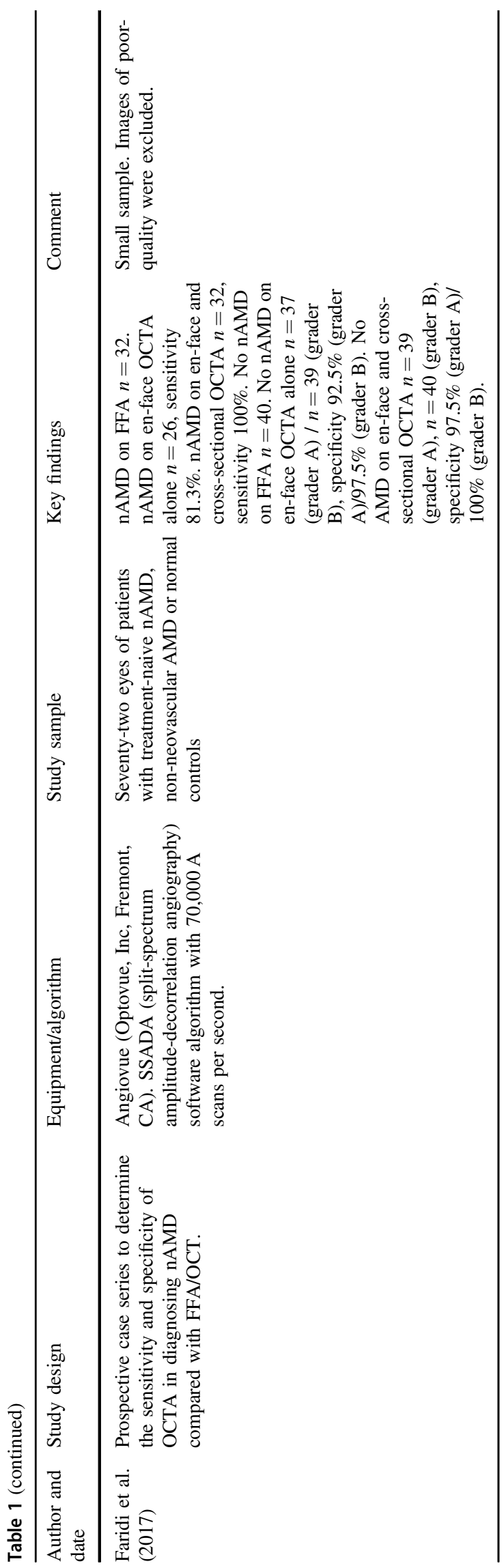

macular degeneration (nAMD). nAMD markedly alters the contours and interfaces between the layers of the outer retina, retinal pigment epithelium and inner choroid through deposition of drusenoid material, the presence of neovascular complexes, exudation of blood and lipid and the development of fibrosis.

The Early Detection of Neovascular Age-Related Macular Degeneration (EDNA) study is a multicentre prospective cohort diagnostic accuracy study assessing the sensitivity and specificity of comparator tests on detection of nAMD in the fellow eye following commencement of Anti-VEGF treatment in the affected eye [3]. The study aims to identify an optimal monitoring regime for early detection of nAMD in the second eye of patients diagnosed with nAMD in one eye. To enable widespread applicability of results, all comparator tests are routinely used in NHS outpatient settings. As many units do not yet have access to OCTA technology, this has not been included as a comparator test in the EDNA study. However, given the increasing evidence suggesting that this emerging technology may in future aid diagnosis and monitoring of nAMD, trainees from the EDNA clinical sites who had been inducted as co principal investigators (co-PIs) were tasked with summarising the current knowledge of OCTA in nAMD.

\section{Methods}

A literature review of EMBASE, MEDLINE and PUBMED databases to cover the period from 01 January 2014 to 31 July 2017 was undertaken. Search terms used were "optical coherence tomography angiography" OR "OCT angiography" OR "OCT-A", AND "neovascular macular degeneration" OR "neovascular age-related macular degeneration" OR "neovascular AMD" OR "nAMD" OR "wet age-related macular degeneration" OR "wet AMD" OR "wet ARMD". The literature review was performed by multiple members of the EDNA Co-PI Writing Group searching the above databases directly or via the NICE Healthcare Databases Advanced Search tool. Only articles published in English and peer reviewed were included. Studies of OCTA in non-neovascular AMD were excluded. Due to the emerging nature of OCTA technology, many published studies include only small numbers of participants, therefore no studies were excluded on the basis of sample size.

Three groups of published material were identified: (a) studies comparing the diagnostic accuracy of OCTA versus FFA and/or ICGA in nAMD with sensitivity and specificity values or positive and negative predictive values (Table 1); (b) studies describing OCTA features of nAMD (Table 2) and (c) review articles and perspectives. 


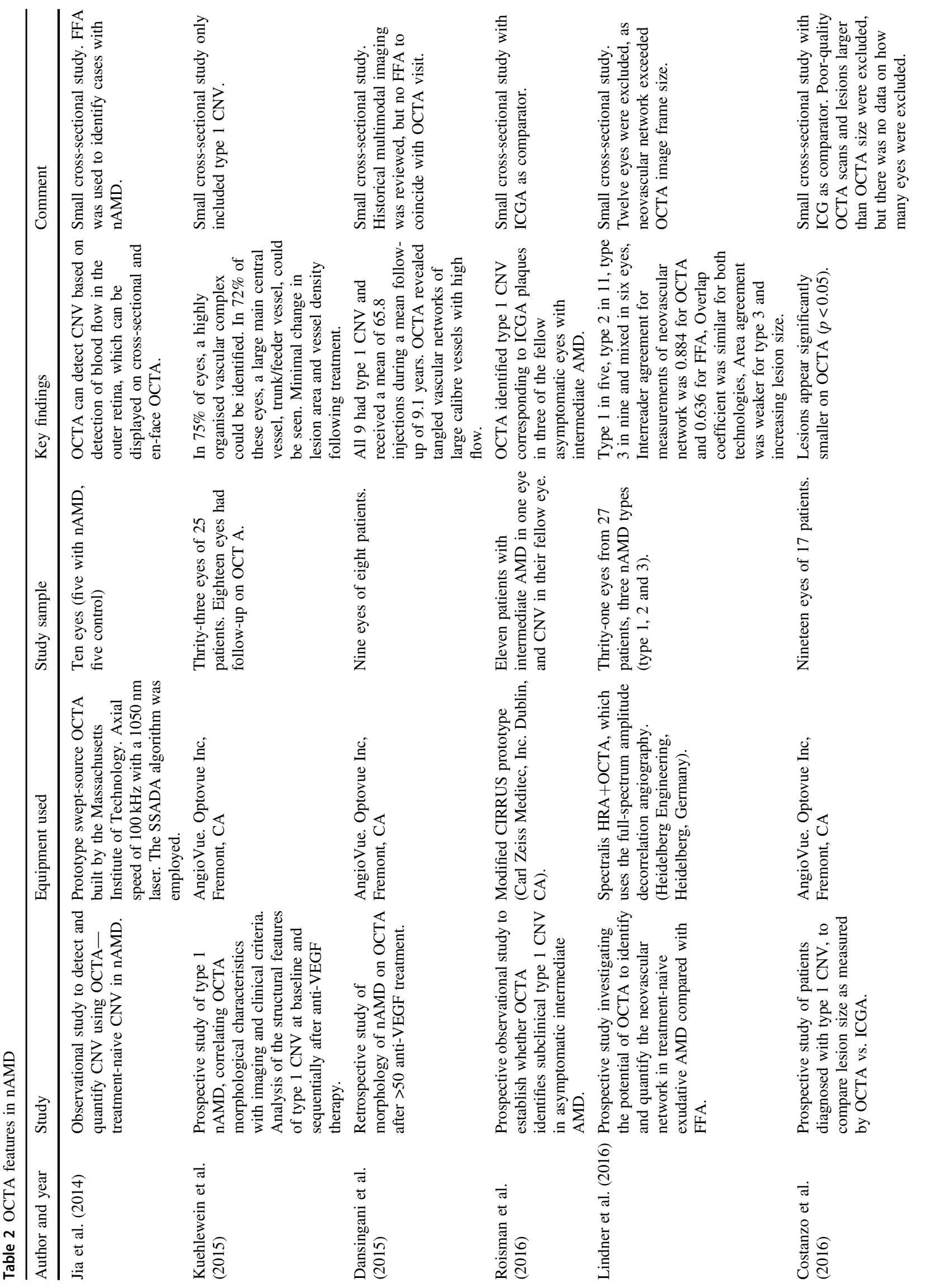




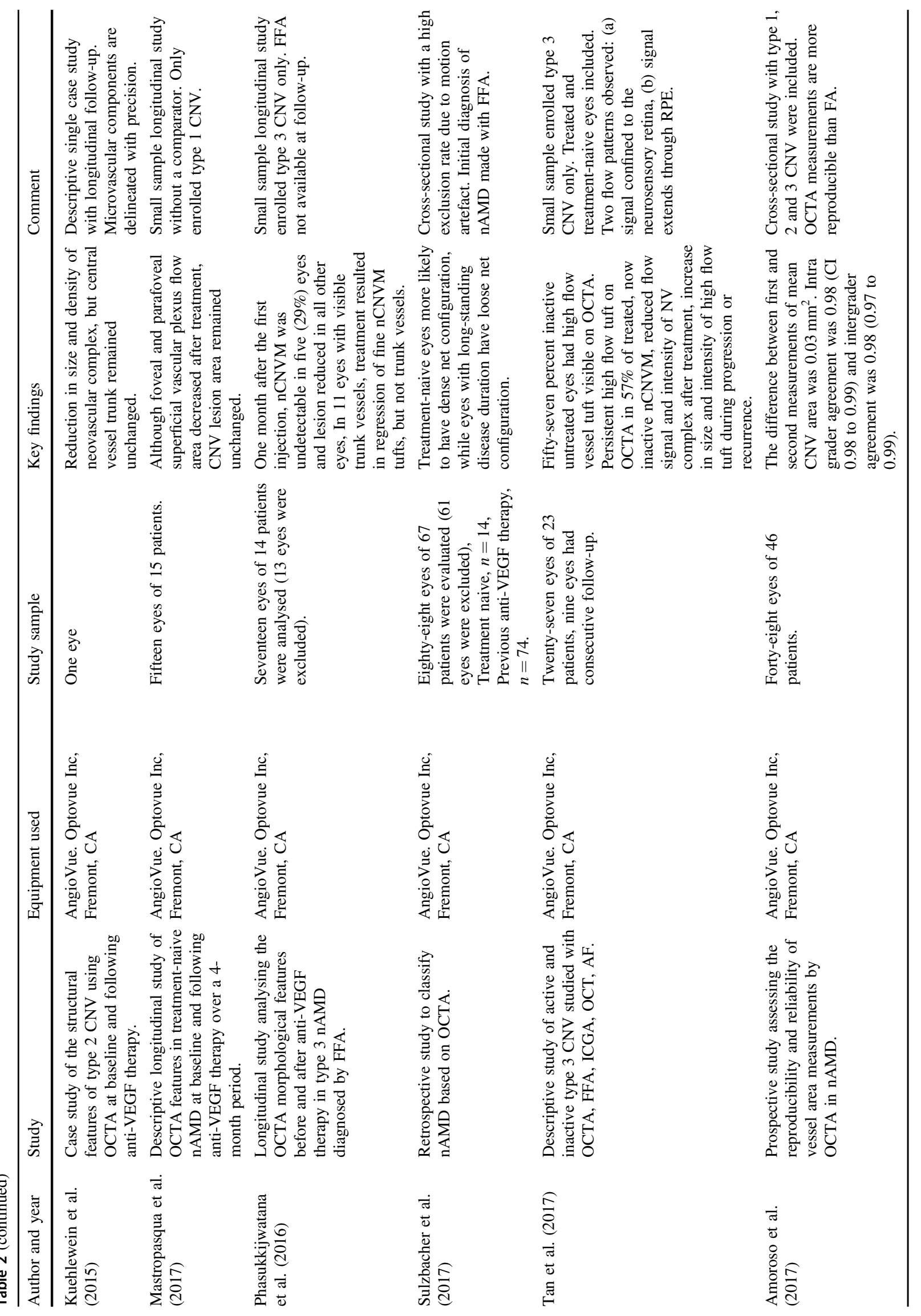


Fig. 1 Forest plots showing sensitivity and specificity of studies, including sample size

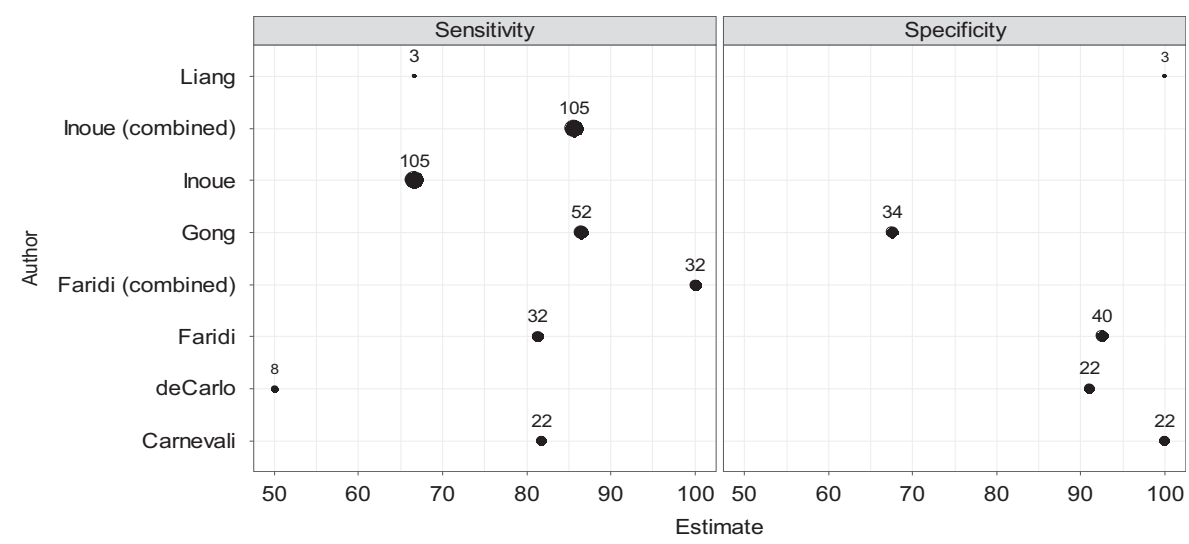

\section{Findings}

\section{Sensitivity and specificity of OCTA in the diagnosis of nAMD}

Six publications providing information on the diagnostic accuracy (sensitivity, specificity or calculations of positive and negative predictive values) of OCTA in nAMD are summarised in Table 1 [4-9]. Most studies compared sensitivity and specificity with the gold standard of FFA and one study used both FFA and ICGA. Figure 1 is a representative forest plot of the sensitivity and specificity of OCTA, and it can be seen that there is only moderate consistency of sensitivity and specificity. The majority of studies had small sample sizes and none reported confidence intervals, thus limiting the accuracy of their results.

De Carlo et al. [4] found the sensitivity to be $50 \%$ in a small study of eight eyes, whilst Gong et al. [5] with a larger sample size of 52 eyes found sensitivity to be $86.5 \%$. In both studies, the reasons identified for false negatives were the presence of tall pigment epithelial detachments and haemorrhage. Liang et al. [6] reported the detection of neovascular complexes on OCTA in patients found to have active leakage on FFA with a sensitivity of $66.7 \%$ and a specificity of $100 \%$. Between these, the reported specificity for OCTA ranged from $67.6 \%$ [5] to $100 \%$ [7]. One study enroled only type $1 \mathrm{CNV}$ and compared the detection of the neovascular complexes by OCTA alone and in combination with structural OCT to the gold standard of FFA [7]. When FFA and OCTA were graded independently, sensitivity was $66.7 \%$ for both. Using en-face OCTA there were six false negative, almost two thirds of which were associated with a large sub retinal haemorrhage. On combining the OCTA information with the cross-sectional structural OCT, the sensitivity for detection of type $1 \mathrm{CNV}$ increased to $85.7 \%$ and specificity was also improved. The authors attribute the false positives to projection artefact, and it would appear that the use of the cross-sectional images helped avoid falsely attributing signals to potentially avascular layers. The higher proportion of eyes in which type $1 \mathrm{CNV}$ was detected suggests that the case mix of nAMD can influence variation in the sensitivity and specificity of detection by OCTA. Conversely, limiting the case mix to a single homogeneous subtype could give rise to falsely high levels of sensitivity. A single study by Gong et al. has provided both positive and negative predictive values for the detection of $\mathrm{CNV}$, and these were estimated to be $80.4 \%$ and $76.7 \%$, respectively [5].

\section{Features of the nAMD lesion on OCTA}

We found 12 publications with descriptions of morphology, and occasionally the size of the neovascular complexes of AMD, with some limiting their study participants to specific subtypes of nAMD (Table 2) [10-21]. Concurrent FFA and or ICGA were infrequently performed, but when available, were used to compare and contrast the features observed on these traditional imaging modalities with those of OCTA [6, 13]. Roisman et al. [14] observed that plaques seen on ICGA correspond to sub-pigment epithelial choroidal neovascular complexes but often without any evidence of active leakage. A small number of studies have measured the area of the CNV complex on OCTA and FFA $[15,16]$. Costanzo et al. [15] showed that the size of the lesion was around 25\% smaller on OCTA compared with FFA. Lindner et al. [17] reported similar overlap coefficients for OCTA and FFA for $\mathrm{CNV}$ lesions, but intergrader agreement on size was higher for OCTA when compared with FFA (0.884 vs. 0.636), indicating better consistency of size determination for the former. However, agreement was poor for OCTA in eyes with retinal angiomatous proliferation (RAP) lesions. Amoroso et al. [18] also reported high intergrader agreement for determination of lesion size using OCTA, but comparison with FFA was not undertaken in this study.

A subgroup of studies [16, 19-21] have observed patients over short follow-up periods, not exceeding 
6 months, and have described changes in the OCTA findings following anti-vascular endothelial growth factor (antiVEGF) therapy. Post-treatment changes that have been noted include regression of peripheral capillary tufts with persistence of central trunk vessels and change from a dense to a loose net configuration of the CNV complexes. However, not all studies are concordant in their views on the change in CNV size, with some reporting reduction in CNV area $[20,21]$, while others have found no change in this parameter although observing a fall in blood flow $[13,18]$.

Many of the studies listed in Table 2 were limited by the exclusion of eyes with lesions that exceeded the OCTA image frame size. Furthermore, eyes with poor-quality OCTA scans were also excluded, and thus the ability of OCTA to detect nAMD in eyes with media opacities and poor fixation remains unevaluated.

Equipment and software algorithms tested in the included studies. Various OCTA systems can use different algorithms to compute and display retinal vessels that have flow [22]. OCTA techniques can be intensity signal based (speckle and phase variance), amplitude-decorrelation (split spectrum angiography; SSADA and full spectrum; FSADA) and complex signal based (optical microangiography; OMAG). Amongst the studies that estimated sensitivity and specificity (Table 1) and in the studies that documented the features of neovascular complexes in AMD, we observed that they were mainly limited to the AngioVue (Optovue Inc, Fremont, CA) and the AngioPlex (Carl Zeiss Meditec. Inc, Fremont, CA), and with only one study using the Heidelberg system that employs the FSADA algorithm.

\section{Discussion}

In contrast to FFA and ICGA, OCTA is an entirely noninvasive method for imaging vascular profiles in the retina and the choroid. It is rapidly gaining popularity both for the detection of nAMD and the subsequent monitoring of the response to treatment [2]. Detecting the flow through blood vessels is the fundamental concept upon which OCTA is based. This principle has been utilised in the detection of normal and abnormal vasculature and is now being applied successfully to the visualisation of CNV in nAMD. OCTA acquisition systems have in-built segmentation algorithms for automated detection of flow and will display vascular profiles by retinal layer, however, none are currently validated, thus expert scrutiny followed by adjustment of the segmentation lines are required. The presence of a CNV on OCTA correlates well with findings on structural OCT and FFA [5]. The sensitivity and specificity values across studies vary (Fig. 1) and appear to be influenced by the both methodology and equipment used, and also by the subtype classification of nAMD. In this context, it is worth noting that type 1 or type $2 \mathrm{CNV}$ appear to be more easily visualised on OCTA compared with RAP or polypoidal complexes.

The increased definition with which the vascular network of a CNV can be visualised on OCTA has led to a better understanding of the structural evolution of these lesions with anti-angiogenic treatment. Despite inactivity on FFA, a vascular network can remain persistent on OCTA [6]. Lesions have been described as exhibiting a central trunk vessel with an extending vascular network. With treatment, these complexes may show a reduction in the overall size, however, the main trunk vessels remain unchanged [13, 16, 19, 21]. Also the changing retinal architecture due to atrophy and or fibrosis can confound the interpretation of lesion size, activity and vascularity based on OCTA. Interestingly, CNV lesions when measured are smaller on OCTA when compared with dyebased angiography [15], but the interobserver coefficient of variation is smaller with the former. This may be due to the better delineation of the margins of the neovascular complex on OCTA resulting in the outlining of a smaller area of involvement as compared with FFA, where leakage can obscure the margins and suggest a greater area of involvement.

The advantages of OCTA are the easy, non-invasive and repeatable quantitative measurement of the dimensions of the vascular network in nAMD. As mentioned previously, interobserver reproducibility in the size measurements of both type 1 and type $2 \mathrm{CNV}$ lesions are better than that made using FFA. Findings on OCTA are concordant with those seen on FFA and ICGA $[8,15,17]$. There is a general consensus on the higher resolution of OCTA in outlining the profiles of the retinal microvasculature, and this attribute has allowed investigators to describe features of the neovascular complexes in AMD that were previously not visible with contrast-based angiography and afforded a better understanding of post-treatment pathophysiology [6].

However, the modality is not without its limitations. Almost all of the studies to date were limited by the sample size, which tended to be small, a lack of negative controls and, in some, the restriction of the study to a single type of nAMD. Even more importantly, no study provided details on the reasons for exclusion such as the presence of media opacities, poor fixation and restricted mobility, which are likely to be commonly encountered in the older population. Therefore, the evidence at present does not fully support the exclusive use of OCTA in the diagnosis of $\mathrm{CNV}$ in the nAMD population.

Vascular leakage cannot be seen using OCTA, as the technology requires flow within the vessels. Furthermore, when vascular pathology results in very fast, very slow or turbid flow, such vessels may not be consistently visualised 
with the current generation of OCTA machines, where the interscan time is fixed. Variable interscan time technology may overcome these obstacles.

There are other limitations that currently exist and reduce the clinical potential of OCTA, many of which have been highlighted by Spaide et al. [23]. The vascular signal can be blocked by overlying pathology $[4,5,8]$, and this may be interpreted as reduced flow within the retinal vascular plexuses or choriocapillaris. Projection artefacts from the superficial or deep retinal plexus vessels can interfere with the signal from the choroidal vasculature. Motion artefacts cause poor-quality scans. False-positive scans have been described when the CNV is visible on OCTA, however the lesions are inactive on FFA, and this finding has been attributed mainly to projection artefact and inaccurate segmentation [15, 23].

The currently available commercial instruments are limited in their scan area. The region of the fundus covered generally lies between $3 \mathrm{~mm} \times 3 \mathrm{~mm}$ and $12 \mathrm{~mm} \times 12 \mathrm{~mm}$. The smaller the field size, the faster the acquisition and the better the resolution. However, the smaller the scan area, the greater the likelihood that a lesion may lie outside of it and areas are missed.

In the majority of the studies that fulfilled the criteria for selection in the present review, we noted that the AngioVue had been used as the OCTA acquisition system. The high frequency of use of the angioVue likely reflected the commercial availability of this instrument, which was the first to be introduced into the market. As the angioVue uses optical microangiography, which is only one of many algorithms deployed in the available instruments, the sensitivity and specificity of other available systems also require validation.

Despite these caveats, our review suggests increasing confidence in the use of OCTA. As more robust evidence accrues, it is likely to become more widely accepted as a form of rapid, sensitive and non-invasive imaging in the detection and management of nAMD.

\section{Disclaimer}

The views and opinions expressed are those of the authors and do not necessarily reflect those of the EDNA programme, NIHR, the UK National Health Service or the Department of Health.

Acknowledgements We wish to acknowledge the support of the EDNA study office based at the University of Aberdeen, with special thanks to Dr Kathryn Bannister. With thanks to David Wright, MRC Innovation Fellow, Health Data Research UK, Centre for Public Health, Queen's University Belfast for generation of the Forest plots.

\section{Compliance with ethical standards}

Conflict of interest Professor Chakravarthy is the Chief Investigator for EDNA and has received honoraria from Zeiss and Heidleberg. EDNA is an National Institute for Health Research (NIHR) funded project; HTA Project: 12/142/07-Early detection of neovascular agerelated macular degeneration. None of the other Authors have any financial disclosures.

\section{References}

1. Cole ED, Ferrara EA, Novis EA, Louzada RN, Waheed NK. Clinical trial endpoints for optical coherence tomography angiography in neovascular age related macular degeneration. Retina. 2016;36:S83-S96.

2. Chalam KV, Sambhav K. Optical coherence tomography angiography in retinal diseases. J Ophthalmic Vis Res. 2016;11:84-92.

3. EDNA; https://www.journalslibrary.nihr.ac.uk/programmes/hta/ 1214207\#/

4. De Carlo TE, Bonini Filho MA, Chin AT, Adhi M, Ferrara D, Baumal CR, et al. Spectral domain optical coherence tomography angiography of choroidal neovascularization. Ophthalmology. 2015;122:1228-38.

5. Gong J, Yu S, Gong Y, Wang F, Sun X. The diagnostic accuracy of optical coherence tomography angiography for neovascular age-related macular degeneration: a comparison with Fundus Fluorescein angiography. J Ophthalmol. 2016. https://doi.org/10. $1155 / 2016 / 7521478$

6. Liang MC, DeCarlo TE, Baumal CR, Reichel E, Waheed NK, Duker JS, et al. Correlation of spectral domain coherence tomography angiography and clinical activity in neovascular age related macular degeneration. Retina. 2016;36:2265-73.

7. Carnevali A, Cicinelli MV, Capuana V, Corvi F, Mazzaferro A, Querques L, et al. Optical coherence tomography angiography: a useful tool for diagnosis of treatment naïve quiescent choroidal neovascularization. Am J Ophthalmol. 2016;169:189-98.

8. Inoue M, Jung JJ, Balaratnasingam C, Dansingani KK, DharamiGavazi E, Suzuki M et al. for the COFT-1 Study group. A comparison between optical coherence tomography angiography for the imaging of type 2 neovascularization. Invest Ophthalmol Vis Sci. 2016;57:OCT314-OCT323.

9. Faridi A, Jia Y, Gao SS, Huang D, Bhavsar KV, Wilson DJ, et al. Sensitivity and specificity of OCT angiography to detect choroidal neovascularization. Ophthalmol Retin. 2017;1:294-303.

10. Jia Y, Bailey ST, Wilson DJ, Tam O, Klein ML, Flaxel CJ, et al. Quantitative optical coherence tomography angiography of choroidal neovascularization in age related macular degeneration. Ophthalmology. 2014;121:1435-44.

11. Kuehlewein L, Bansal M, Lenis TL, Iafe NA, Sadda SR, Bonini Filho MA, et al. Optical coherence tomography angiography of type 1 neovascularization in age related macular degeneration. Am J Ophthalmol. 2015;160:739-48.

12. Dansingani KK, Freund KB. Optical coherence tomography angiography reveals mature, tangled vascular networks in eyes with neovascular age-related macular degeneration showing resistance to geographic atrophy. Ophthalmic Surg Laser Imaging Retin. 2015;46:907-12.

13. Tan A, Dansingani KK, Yannuzzi LA, Sarraf D, Freund KB. Type 3 neovascularization imaged with cross-sectional and enface optical coherence tomography angiography. Retina. 2017;37: 234-46. 
14. Roisman L, Zhang Q, Wang RK, Gregori G, Zhang A, Chen CL, et al. Optical coherence tomography angiography of asymptomatic neovascularization in intermediate age related macular degeneration. Ophthalmology. 2016;123:1309-19.

15. Costanzo E, Meire A, Quereques G, Capuano V, Jung C. Soied. Type 1 choroidal neovascularization lesion size: Indocyanine green angiography versus optical coherence tomography angiography. Invest Ophthalmol Vis Sci. 2016;57:OCT307-OCT313.

16. Phaskkijwatana N, Tan ACS, Chen X, Freund KB. Sarraf. Optical coherence tomography angiography of type 3 neovascularization in age related macular degeneration after antiangiogenic therapy. Br J Ophthalmol. 2017;101:597-602.

17. Linder M, Fang PP, Steinberg JS, Domdei N, Pfau M, Krohne TU, et al. OCT Angiography-based detection and quantification of the neovascular network in exudative AMD. Invest Ophthalmol Vis Sci. 2016;57:6342-8.

18. Amoroso F, Miere A, Semoun O, Jung C, Capuano V, Souied EH. Optical coherence tomography angiography reproducibility of lesion size measurements in neovascular age related macular degeneration (AMD). Br J Ophthalmol. 2017. https://doi.org/10. 1136/bjophthalmol-2017-310569

19. Mastropasqua L, Toto L, Borrelli E, Carpineto P, Antonio LD, Mastropasqua R. Optical coherence tomography angiography assessment of vascular effects occurring after aflibercept intravitreal injections in treatment naïve patients with wet age related macular degeneration. Retina. 2017;37:247-56.

20. Sulzbacher F, Pollreisz A, Kaider A, Kickinger S, Sacu S, SchmidtErfurth U, on behalf of the Vienna Eye Study Center. Identification and clinical role of choroidal neovascularization characteristics based on optical coherence tomography angiography. Acta Ophthalmol. 2017;95:414-20.

21. Kuehlewein L, Sadda SR, Sarraf D. OCT angiography and sequential quantitative analysis of type 2 neovascularization after ranibizumab therapy. Eye. 2015;29:932-5.

22. Chen CL, Wang RK. Optical coherence tomography based angiography. Biomed Opt Express. 2017;8:1056-82.

23. Spaide RF, Fujimoto JG, Waheed NK. Image artefacts in optical coherence angiography. Retina. 2015;35:2163-80. 